\title{
MEASURING INNOVATION EFFECTIVENESS BY MEANS OF A CONCEPTUAL TEST OF ELECTRICITY AND MAGNETISM
}

\author{
A. Vidaurre, J. Riera, J.M. Meseguer-Dueñas, J. Molina Mateo, J.A. Gómez- \\ Tejedor, I. Tort-Ausina, M.A. Gámiz-González \\ Universitat Politècnica de València (SPAIN)
}

\begin{abstract}
For this work, we report on the results of the BEMA assessment for students of Electricity courses of Engineering Degrees taught at the Higher Technical School of Design Engineering (ETSED) Universitat Politècnica de València (UPV). The BEMA pre-test and post-test assessment was delivered at the beginning and at the end of the course to students of the Degrees on Electronic Engineering and Industrial Automation (DEEIA) and Aerospace Engineering (DAE). The gain in the performance of BEMA test was analysed for both curricula and for three categories: electrostatics, dc current circuits and magnetostatics. The results show better results for DAE curriculum in the pre-test, but a lower gain in the post-test. The highest gain is obtained in magnetostatics, and no influence of the use of screencast is appreciated.
\end{abstract}

Keywords: Innovation effectiveness, conceptual test, gain.

\section{INTRODUCTION}

Higher Education is under a continuous transformation process. The advances in the field of Information and Communications Technology provide new tools that support the academic needs in different teaching and learning models. Flip teaching, blended learning, teamwork, effective oral and written communication; problem-solving strategies and peer assessment are, among others, methodologies that are being used in our daily instruction model.

In order to quantify student-learning gains after the introduction of a methodological change, standardized assessments in introductory physics (i.e., concept inventories) have been proposed. The use of these assessments to evaluate student learning is only productive if they are aligned with our learning goals. From our point of view, the concept inventory Brief Electricity and Magnetism Assessment (BEMA) is well aligned with the topics and content depth of our Electricity courses [1]. BEMA includes basic electrostatics, circuit's analysis, magnetic fields and forces, and induction. The core ideas that are potentially assessed by BEMA are: interactions can cause changes in motion and interactions are mediated by fields [2]. There are others instruments to asses student's understanding of Electricity and Magnetism concepts, such as Conceptual Survey of Electricity and Magnetism CSEM [3]. With the exception of electric circuits, omitted by the CSEM, both instruments cover similar topics; in fact, several items are common to both tests. Recent work has shown that the CSEM and BEMA are equivalent when measuring changes in pedagogy [4].

There are several methods to measure student's outcomes when comparing pretest and postest results. The most popular metric used in physic education research is the normalized gain, $g$, developed by Hake [5] and defined as the ratio between the actual average gain to the maximum possible average gain. Another method, that is commonly use is to calculate the normalized gain for each student, $g_{l}$, and then average the normalized gains to calculate $g$ for the whole group [6], [7]. Using individual gains provides more detailed information than the average value.

Nowadays, the interest of video as a tool in the learning process has increased [8]. Videos can explain concepts, which are difficult to explain with tables, figures or books, and it improves students' understanding. In the flip teaching methodology, video is frequently used as a pedagogical tool to provide students resources to prepare the contents before the class. It has been showed that it provides positive cognitive results like effectiveness and satisfaction in the students [9]. Many studies have been focused on analyzing the pedagogical results in students learning that provided video as learning tools, e.g., Zhang et al. studied effectiveness in e-learning video [10]. Tort-Ausina et al. found that most of students prefer the video format to the written document [11]. This result is confirming what seems to be a general social trend for young people, which usually prefer to see and listen than to read. 
For this work, we report on the results of the BEMA assessment for students of Electricity courses of Engineering Degrees taught at the Higher Technical School of Design Engineering Universitat Politècnica de València (UPV). Two different curricula are evaluated: a second semester traditional curriculum in which have combined the use of traditional textbooks in some of the units and screencast in others and a 6 weeks intensive course in which particular emphasis has been placed in the vector field concept as wells as Maxwell laws.

\section{METHODOLOGY}

This paper is focused on measuring the gain in the post-test BEMA in comparison to the pre-test. The BEMA pre-test and post-test assessment were delivered to students at the beginning and at the end of the course to students of the Degrees on Electronic Engineering and Industrial Automation (DEEIA). In the case of Degree on Aerospace Engineering (DAE) the tests were delivered just before and after the 6 weeks when electromagnetism part was taught.

In the case of Electricity for DEEIA Degree, some of the classes follow a traditional lecturer scheme whereas some others follow a flip teaching (FT) model. In the last case, students were asked to watch videos and perform some readings and solve some tests at home. In some particular units: electrostatic and direct current circuits, the use of videos was mostly recommended whereas in the case of magnetism no videos were provided to students and only the text book was recommended. The course also include a unit of alternating current circuits, but BEMA test does not includes any question about this topic.

Regarding to DAE, the electromagnetism is part of the Physics course that also includes mechanics, fluids, and thermodynamics. As the students have been working more deeply on field theory, the electromagnetism starts introducing the Maxwell laws and then their consequences: electrostatic, direct current circuits and magnetostatics. Apart from this, the pedagogical aspects of both the DEEIA and DAE were quite similar.

The BEMA test was administrated following the instructions: time limit $45 \mathrm{~min}$, the same small incentive for all students who completed the test regardless of the score. Only students who completed the pre-test and post-test have been considered; they were 79 from a total of 154 students in the case of DEEIA and 42 from 78 students in DAE. The BEMA items have been divided into three categories covering the topics: electrostatics, dc circuits, and magnetostatics [12]. Using these categories, we compared DEEIA and DAE performance in each category. It was also possible to compare the effect of the use of videos as part of the resources, in the case of DEEIA.

The average in understanding increase is measured by the average percentage gain, $\mathrm{G}=$ Post-Pre for each student. Then the average gain can be obtained for each degree, as well as the average gain in each category. We define the difference in performance between the two degrees as $\triangle G=G_{D A E}-G_{D E E I A}$. In the same way, we can calculate the difference in performance in each category or item. A positive (negative) $\Delta G$ corresponds to an item where, on average, the DAE students performed better (worst) than DEEIA students.

We are also interested in finding out if the screencast on specific topics that are delivered to students, have some effect on their knowledge about the topic. In half of the units, including electrostatic and dc circuits, students used traditional written documents: books, slides; in the other half of the units, they could watch videos prepared by the teachers. The units have been selected in such a way that both parts have similar difficulty, according to our experience. If there is some difference in the average gain of both parts, it could be attributed to the effect of the use of the videos.

\section{RESULTS}

\subsection{Individual and average BEMA scores}

Fig. 1 shows the BEMA pre-test and post-test scores, out of 10 , for each student of both degrees. It can be seen that the majority of students fall over the straight-line $y=x$, which means that the majority of students improve their results, as one could expect. However, there are a significant number of students, which obtain worst results in the post-test than in the pre-test (8 of DAE and 20 of DEEIA). This could be attributed to a lack of concentration during answering the test. It can also be observed that there is a great dispersion in the results. 


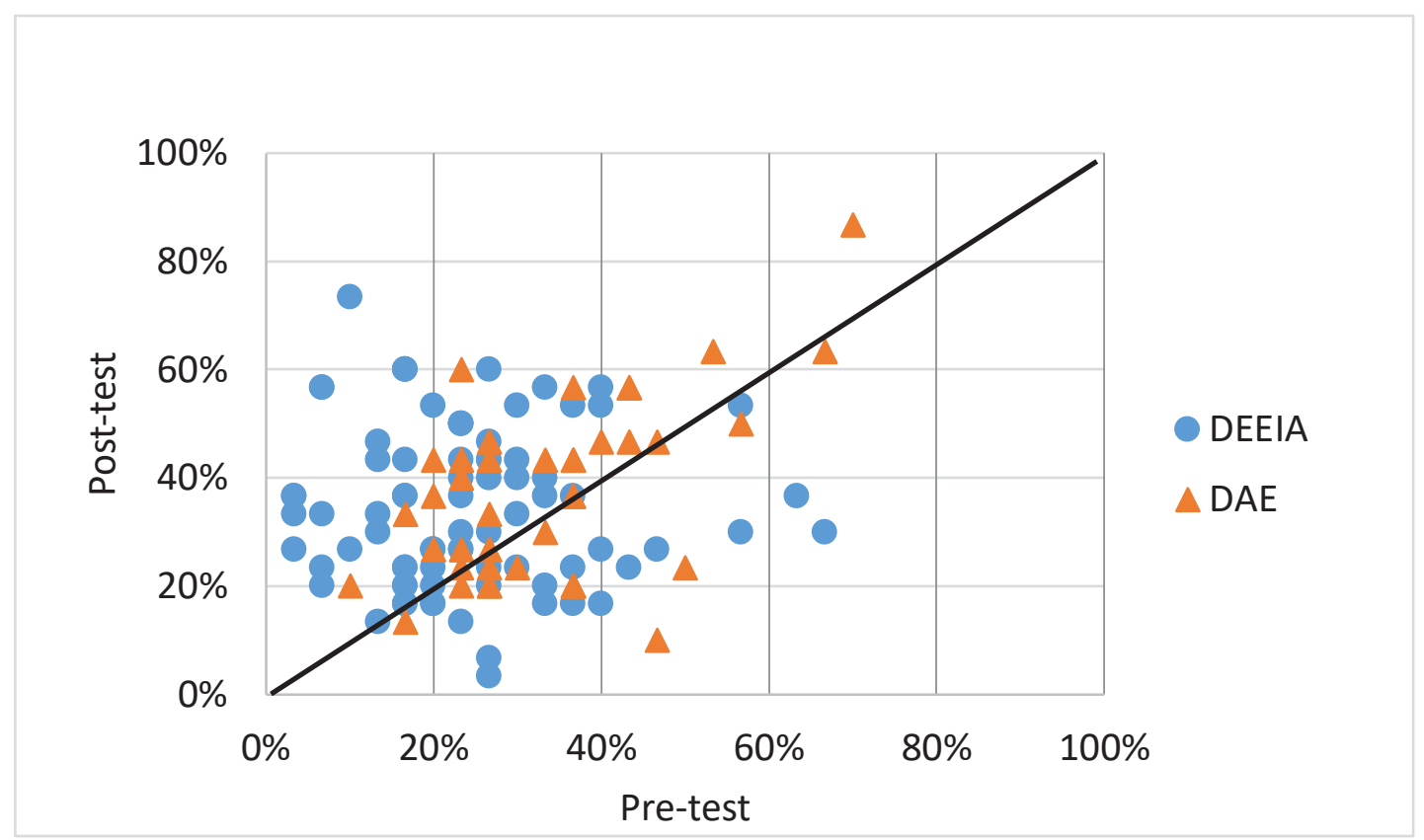

Figure 1. Post-test score as a function of the pre-test score for DAE and DEEIA Degrees.

The straight-line represents students who obtain the same score in both tests

Table 1 presents the average score, as well as the standard deviation for both curricula. While the results of the pre-test are slightly higher in the DEEIA curriculum, the results of the post-test are better for the DAE curriculum indicating a higher gain in the last. All the results show a high value of the standard deviation, as correspond to the data showed in Fig. 1 . The average gain, G, is $10 \%$ for DEEIA and $3.5 \%$ for $D A E$, and $\Delta G=G_{D A E}-G_{D E E I A}=-6.5$. The corresponding normalized gain, $g$, calculated from the average gain divided by the maximum gain (100-G), is 0.13 for DEEIA and 0.065 for DEA, both falling in the "low g" courses, according to the Hake classification [5].

Table 1. Average pre and post-test scores, and standard deviation.

\begin{tabular}{l|c|c|c|c}
\hline \hline & $\begin{array}{c}\text { Average pre-test } \\
(\%)\end{array}$ & sd & $\begin{array}{c}\text { Average post-test } \\
(\%)\end{array}$ & sd \\
\hline DEEIA & 24.4 & 13.2 & 34.4 & 14.9 \\
\hline DAE & 34.1 & 13.7 & 38.4 & 16.4 \\
\hline
\end{tabular}

\subsection{BEMA scores by category}

The BEMA items have been divided into three categories covering the topics: electrostatics, dc circuits and, magnetostatics. Fig. 2 shows the pre-test score for each category and for both curricula. It can be observed that for both curricula the best results correspond to electrostatics, followed by dc current and magnetostatics. The results are always higher for DAE, what could be explained by the moment in which the test has been administrated (later in DAE, after the topic of field's theory have been lectured. From these results, it can be concluded that DAE starts with higher knowledge of electricity and magnetism. 


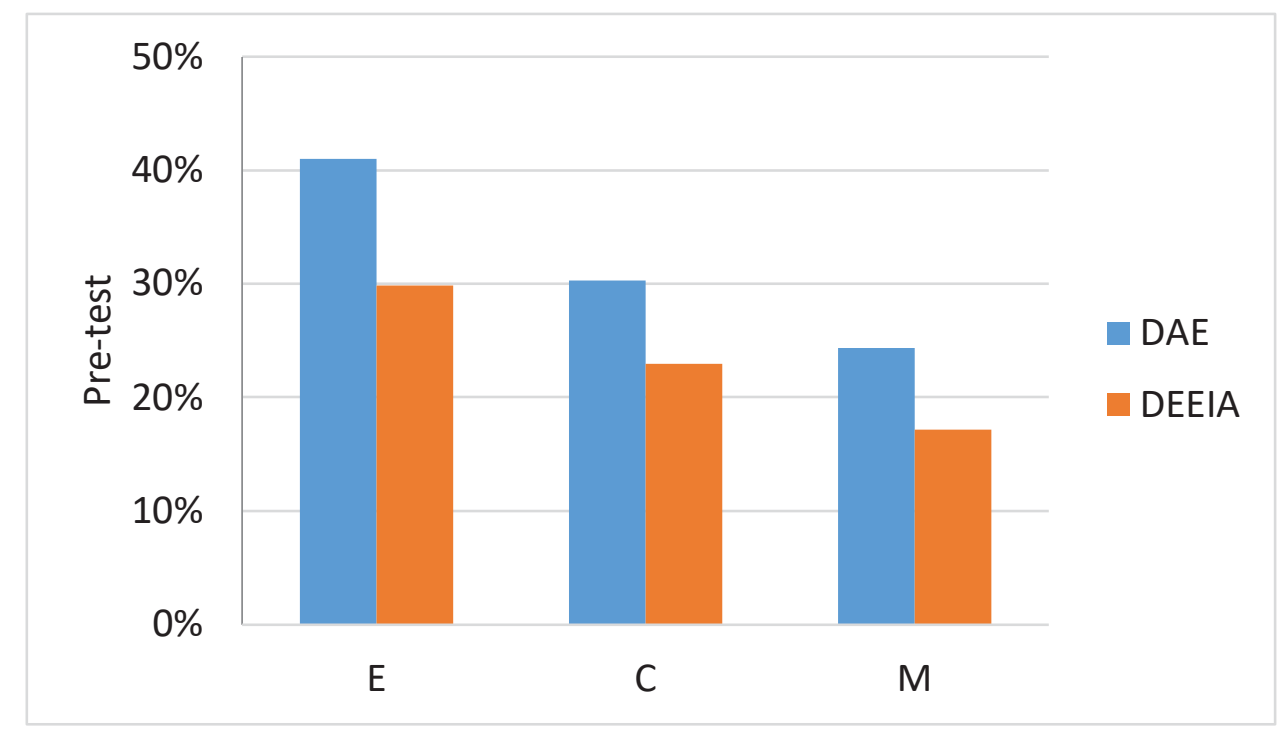

Figure 2. Pre-test score for the tree categories and for DAE and DEEIA Degrees. E: electrostatics, C; dc current, and M: magnetostatics.

The corresponding results for the post-test are shown in Fig. 3. Once again, the results are better for DAE in all categories, although showing minor differences in electrostatics and magnetostatics and no difference in dc current. This result can be understood by the lower capacity of improvement of DAE curriculum, as they spend less lecturer time and starts with a higher BEMA score.

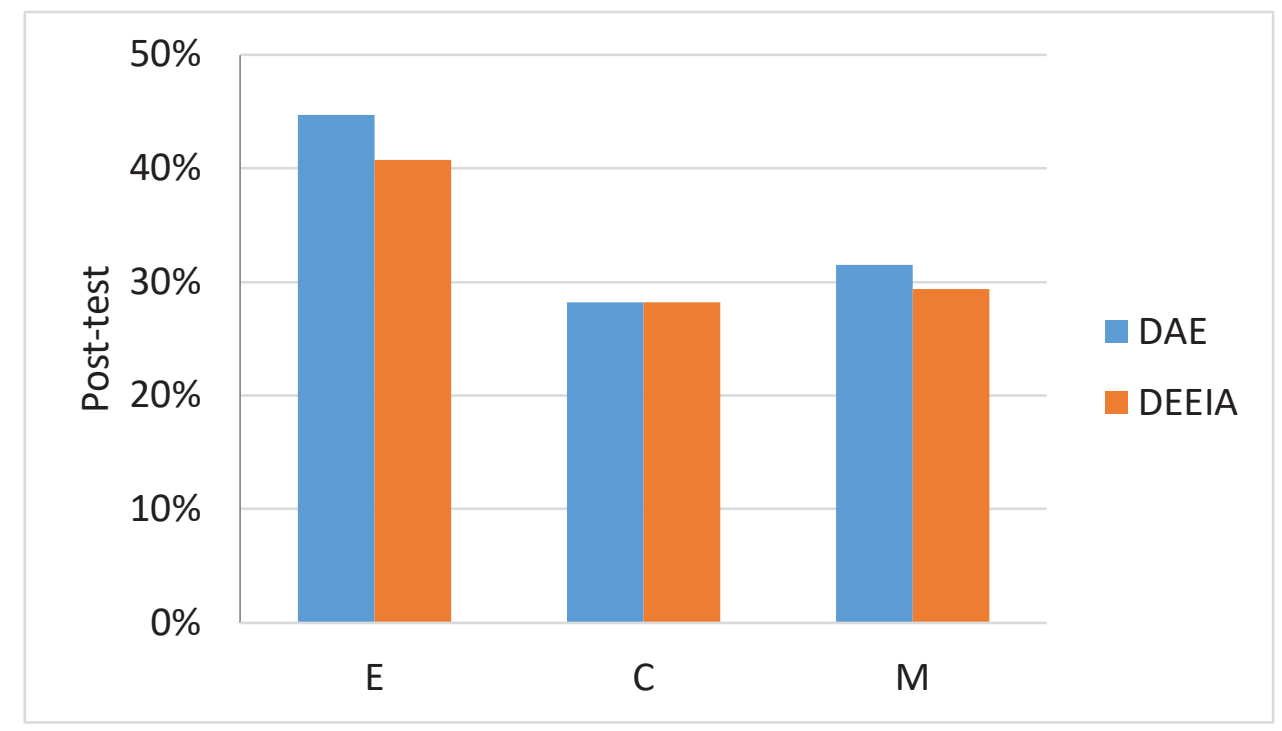

Figure 3. Post-test score for the tree categories and for DAE and DEEIA Degrees. E: electrostatics, C; dc current, and M: magnetostatics.

Fig. 4 show the normalized gain, calculated as the average of the normalized gain of each student. The maximum gain is obtained in magnetostatics whereas the lower gain is obtained for dc current circuits for both curricula; getting even a negative gain in DAE curriculum. One possible explanation is the higher start point in the category of electrostatics and a lack of motivation in getting the correct answer in the post-test. it should be taken into account that the BEMA test has an excessive number of options, as has been mentioned in the literature, and also that students were only given extra points for completing the exam.

Regarding to the influence of complementary resources (screencast) in performing the BEMA test, the results did not show a clear effect. In the case of DEEIA curriculum, magnetostatics was taught without this resource and is the category in which the gain is higher (see Fig. 4). In the case of DAE screencast were not used and the results are similar (higher gain in magnetostatics). 


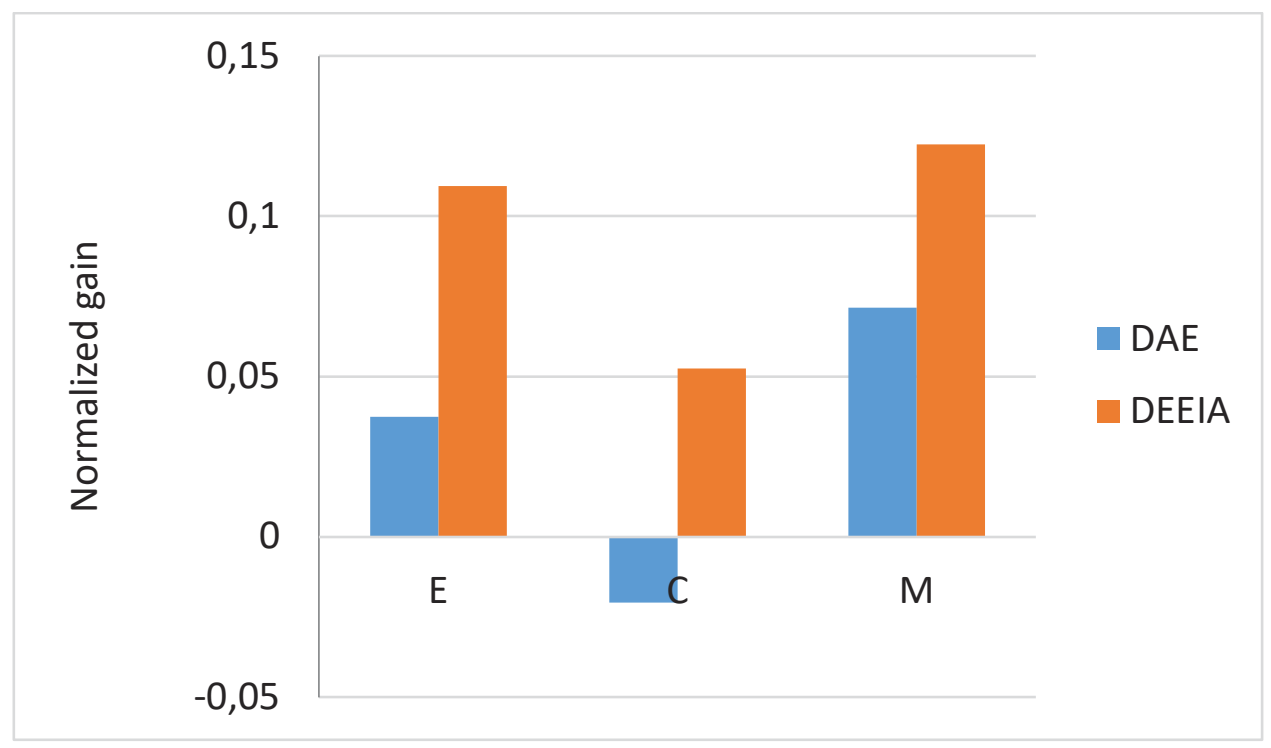

Figure 4. Normalized gain, g, for the tree categories and for DAE and DEEIA Degrees.

E: electrostatics, C; dc current, and M: magnetostatics.

If the analysis is performed by items, we found that the item number 1 , which ask about the relationship of the Coulomb force with distance, $90 \%$ of students give the correct answer. By contrast, almost no student give the correct answer to item number 14, in which they are asked about the electric field created by a solenoid. Probably they misunderstood the question because they relate the solenoid with the magnetic field.

Comparing the BEMA results of both curricula with the corresponding to other Universities that follow a similar instruction on electricity and magnetism (Carnegie Mellon University, Georgia Institute of Technology, North Carolina State University, and Purdue University [12]) we obtain a close or even higher result for the pre-test. The average pre-test ranging from $22 \%$ to $28 \%$, in their case, while we have obtained 24.4 and 34.1 for DEEIA and DAE curricula, respectively. However, they obtain much better performance in the BEMA post-test, mainly in the M\&I curriculum, getting BEMA scores higher that $50 \%$ in many cases whereas we obtain values lower than $40 \%$ in both curricula.

\section{CONCLUSIONS}

In this paper, the gain in the BEMA test was analysed in two different curricula. DEEIA is a common second semester course. The time lecture and the syllabus are also the most commonly taught. DAE curriculum corresponds to a 6 weeks intensive course, part of a Physics annual course, with more emphasis on fields and Maxwell's equations. The gain in the BEMA performance is compared for both curricula. It is obtained that DAE students obtained a higher score in the BEMA pre-test than DEEIA students. By contrast, the gain was lower in the DAE course. The same results were found when the gain of the tree categories were calculated. The highest gain was obtained for magnetostatics and the lowest for dc current where DAE got a negative gain. This fact is very difficult to explain and more research is needed. One possibility is try to increase the motivation of students in getting better results.

No differences in performance of the BEMA test have been found when students have been provided of complementary resources as screencast. Although they watched them and when they are asked their opinion is very positive, it seems that it must be other factors that produce more effect in the BEMA performance.

\section{ACKNOWLEDGEMENTS}

Authors would like to thank the Institute of Education Sciences of the Universitat Politècnica de València (Spain) for supporting the Teaching Innovation Group e-MACAFI and for the financial support through PIME Project PIME/2018/A25 and PIME Project PIME/2018/A26. 


\section{REFERENCES}

[1] L. Ding, R. Chabay, B. Sherwood, and R. Beichner, "Evaluating an electricity and magnetism assessment tool: Brief electricity and magnetism assessment," Phys. Rev. Spec. Top. - Phys. Educ. Res., vol. 2, no. 1, pp. 1-7, 2006.

[2] J. T. Laverty and M. D. Caballero, "Analysis of the most common concept inventories in physics: What are we assessing?," Phys. Rev. Phys. Educ. Res., vol. 14, no. 1, p. 10123, 2018.

[3] D. P. Maloney, T. L. O'Kuma, C. J. Hieggelke, and A. Van Heuvelen, "Surveying students' conceptual knowledge of electricity and magnetism," Am. J. Phys., vol. 69, no. S1, pp. S12-S23, 2001.

[4] S. J. Pollock, "Comparing student learning with multiple research-based conceptual surveys: CSEM and BEMA," AIP Conf. Proc., vol. 1064, no. October, pp. 171-174, 2008.

[5] R. R. Hake, "Interactive-engagement versus traditional methods: A six-thousand-student survey of mechanics test data for introductory physics courses," Am. J. Phys., vol. 66, no. 1, pp. 64-74, 1998.

[6] L. Bao, "Theoretical comparisons of average normalized gain calculations," Am. J. Phys., vol. 74, no. 10, pp. 917-922, 2006.

[7] J. Von Korff et al., "Secondary analysis of teaching methods in introductory physics: A $50 \mathrm{k}$ student study," Am. J. Phys., vol. 84, no. 12, pp. 969-974, 2016.

[8] A. M. F. Yousef, M. Amine Chatti, and U. Schroeder, Video-Based Learning: A Critical Analysis of The Research Published in 2003-2013 and Future Visions. 2014.

[9] G. C. Gannod, J. E. Burge, and M. T. Helmick, "Using the inverted classroom to teach software engineering," in Proceedings of the 13th international conference on Software engineering - ICSE '08, 2008, p. 777.

[10] D. Zhang, "Interactive Multimedia-Based E-Learning: A Study of Effectiveness," Am. J. Distance Educ., vol. 19, no. 3, pp. 149-162, Sep. 2005.

[11] I. Tort-Ausina et al., "Analysis of the use of videos as part of flip teaching in laboratory sessions in Engineering Degrees," Proc. ICERI2017 Conf. 16th-18th Novemb. 2017, Seville, Spain, no. November, pp. 4783-4789, 2017.

[12] M. A. Kohlmyer et al., "Tale of two curricula: The performance of 2000 students in introductory electromagnetism," Phys. Rev. Spec. Top. - Phys. Educ. Res., vol. 5, no. 2, p. 020105, 2009. 